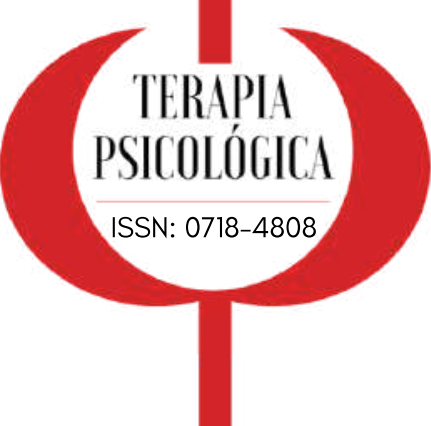

\title{
Metacognición, apego y sintomatología en esquizofrenia primer episodio
}

\section{Metacognition, attachment and symptomatology in first-episode schizophrenia}

\author{
Sergio Vergara-Ramírez \\ (iD) 0000-0002-8072-7327 \\ vergarasergiolegmail.com \\ Universidad Católica del Maule, \\ Facultad de Medicina, Departamento \\ de Ciencias Clínicas, Talca, Chile. \\ Médico Psiquiatra.
}

\author{
Alejandro León-Uribe \\ (D) 0000-0002-3933-8215 \\ alejo.leonegmail.com \\ Universidad de Santiago de Chile, \\ Dirección de Postgrados, Facultad de \\ Ciencias Médicas, Santiago, Chile \\ Psicólogo Clínico.
}

\section{Resumen:}

Los sujetos con Esquizofrenia experimentan menoscabo en la capacidad para formar ideas complejas sobre sí mismos y otros, definido como déficits metacognitivos. El presente estudio evaluó las correlaciones entre metacognición, apego y síntomas en pacientes con Esquizofrenia primer episodio en el Hospital Regional de Talca entre 2016 y 2018. Se diseñó un estudio cuantitativo, relacional, prospectivo, transversal y observacional. Treinta y un individuos fueron evaluados utilizando la Metacognitive Assessment ScaleAbbreviated para metacognición. También se evaluó apego y sintomatología. Se encontraron bajos puntajes metacognitivos y la mayoría de los participantes fueron catalogados como apego evitante. Menores capacidades metacognitivas se correlacionaron significativamente con mayor sintomatología negativa y de desorganización. Conclusiones. Nuestros resultados respaldan la inclusión de los déficits metacognitivos y patrones de apego en los modelos de Esquizofrenia, lo que podría mejorar nuestra comprensión de los síntomas negativos y de desorganización. Además, se discute sobre su aporte al desarrollo de intervenciones psicoterapéuticas en Esquizofrenia desde el primer episodio.

Palabras Clave: Metacognición, Apego, Sintomatología, Esquizofrenia

\section{Abstract:}

Subjects with Schizophrenia experience decrements in their ability to form complex ideas about themselves and others, defined as metacognitive deficits. The current study assessed correlations between metacognition, attachment and symptoms in patients with first-episode Schizophrenia. A quantitative, relational, prospective, cross-sectional and observational study was designed. Thirty-one individuals were assessed using the Metacognitive Assessment Scale-Abbreviated for metacognition. Attachment and symptomatology were also measured. Results. Low metacognitive scores were found, and most participants were classified as dismissing attachment. Lower metacognitive abilities were significantly correlated with greater negative and disorganized symptoms. Conclusions. Our results support the inclusion of metacognitive deficits and attachment patterns in Schizophrenia models, which could improve our understanding of negative and disorganized symptoms. In addition, their contribution to the development of psychotherapeutic interventions in Schizophrenia from the first episode is discussed.

Keywords: Metacognition, Attachment, Symptomatology, Schizophrenia. 


\section{Introducción}

En su modelo de la Esquizofrenia, originalmente publicado en 1911, Bleuler (2011) planteó que este trastorno es en parte el resultado de una disrupción de los procesos asociativos necesarios para formar ideas integradas sobre sí mismo y los demás, que estarían a la base de la actividad humana más compleja y significativa. La alteración de estos procesos ha sido destacada en los últimos años junto a otras disfunciones, como la desorganización narrativa, la presencia de esquemas y ciclos interpersonales disfuncionales, las alteraciones en la intersubjetividad y los déficits metacognitivos (Salvatore et al., 2017). Se ha señalado que las personas que padecen Esquizofrenia presentan graves dificultades en darse cuenta de los estados mentales propios y ajenos, utilizándose el término metacognición para referirse a la capacidad de representarse dichos estados mentales (Lysaker et al., 2014b). El concepto metacognición fue definido inicialmente por Flavell (1979) como "cognición de la cognición". Desde una perspectiva clínica, el término se ha introducido en el campo de los trastornos de la personalidad y otros trastornos mentales graves. En este ámbito, para Semerari (2002), metacognición se refiere al aspecto del procesamiento de la información que monitorea, interpreta, evalúa y regula los contenidos y procesos de su organización. De acuerdo con Lysaker et al. (2014a), metacognición se relaciona con la capacidad necesaria para formar, revisar y reformular ideas de lo que es creído, sentido, soñado, temido, aparentado o pretendido, tanto por uno mismo como por otros, que describen una diversa gama de actos cognitivos internos y determinados socialmente. A partir de esto, se ha establecido una capacidad metacognitiva global conformada por cuatro dominios metacognitivos: Autorreflexividad, habilidad para tomar conciencia de los estados mentales de uno mismo; Comprensión de la mente de Otros, la habilidad para pensar acerca de los estados mentales de los demás; Descentración, la capacidad de adoptar una perspectiva no egocentrista y así poder interpretar los acontecimientos sin ligarlos estrictamente a nosotros y finalmente, Maestría, la habilidad para establecer estrategias útiles que permitan lidiar y resolver conflictos psicológicos o la angustia relacionada con ellos (Lysaker, Buck y Hamm, 2016). Cada dominio presenta sus propias subfunciones, las que expresan niveles progresivamente más complejos de esa capacidad evaluada, organizadas jerárquicamente (Lysaker et al., 2016, Semerari, 2002, Semerari et al., 2003). Los dominios Autorreflexividad, Comprensión de la mente de otros y Maestría tienen una subfunción basal denominada Requisitos Básicos, que se refiere a la habilidad para reconocer la mente propia y ajena como algo autónomo, separado y diferente de lo que es la mente de otros, siendo capaz de definir de modo plausible los términos de un problema (Semerari, 2002). Además de los Requisitos Básicos, para el caso de los dominios Autorreflexividad y Comprensión de la mente de otros, se consideran las 
subfunciones de Identificación, Relación entre las Variables, Diferenciación e Integración. Las subfunciones de Identificación y Relación entre las Variables permiten la monitorización de las cogniciones y emociones hipotetizando relaciones de causalidad inmediata con variables internas $\circ$ externas. La subfunción Diferenciación permite conocer la naturaleza representacional e hipotética del pensamiento distinguiendo entre realidad interna y externa. Por último, la subfunción Integración permite la construcción de narrativas coherentes para describir el escenario mental propio y ajeno a través de la evolución de un estado mental o la integración entre estados diferentes. El dominio Descentración no presenta subfunciones. Para el dominio Maestría, además de los Requisitos Básicos, se suman las estrategias de primer, segundo y tercer nivel, en orden de aplicar medidas más complejas para resolver problemas (Semerari, 2002). En el caso de las personas con Esquizofrenia, éstas presentan capacidades metacognitivas menores en relación a la población general y los afectados por otros trastornos psiquiátricos (Tas, Brown, Aydemir, Brüne y Lysaker, 2014, Lysaker et al., 2018). Existe evidencia de la correlación entre déficits metacognitivos y mayor sintomatología, principalmente síntomas negativos y de desorganización en pacientes con Esquizofrenia de larga data (Hamm et al., 2012) y también en pacientes que cursan un primer episodio (MacBeth et al., 2014, Vohs et al., 2014, Trauelsen et al., 2016). No tan solo se ha visto correlación de metacognición con la sintomatología negativa concurrente, sino también sería un factor de riesgo para la aparición de ella al cabo de seis meses (Hamm et al., 2012). Por otra parte, se ha documentado la asociación de los déficits metacognitivos en Esquizofrenia con alteraciones neurocognitivas, como memoria verbal y visual, velocidad de procesamiento, nivel de inteligencia premórbida (Lysaker et al., 2005, Nicolò et al., 2012), baja capacidad de insight (Vohs, George, Leonhardt y Lysaker, 2016) y sacar conclusiones apresuradamente (Buck, Warman, Huddy y Lysaker, 2012). Además, se ha encontrado correlación con menor funcionamiento social de manera directa (Lysaker et al., 2010a, Lysaker et al., 2011) e indirectamente mediando el impacto de neurocognición sobre calidad y cantidad de relaciones sociales (Lysaker et al., 2010b) y con alianza terapéutica (Davis, Eicher y Lysaker, 2011).

Otra de las variables que se estudiará corresponde al apego. La teoría del apego desarrollada por Bowlby, una de las más influyentes en la historia de la Psicología, ha permitido conceptualizar la propensión de los seres humanos a stablecer intensos vínculos significativos con otras personas a lo largo de todo el ciclo vital y explicar las múltiples formas de padecimiento emocional, incluyendo la ansiedad, ira, depresión, desapego emocional a que dan origen la separación y la pérdida sufridas (Lecannelier, 2012). La sintonía del cuidador a las conductas de apego del niño (llanto, sonrisa, etc.) y respuestas confiables a las señales de estrés en el niño, van generando en él la sensación de seguridad con el cuidador. 
Dicha seguridad en el apego contribuye al desarrollo temprano de la capacidad de enlazar la conducta con estados mentales, sean sentimientos, pensamientos y/o deseos. Por el contrario, una menor sintonía del cuidador con las señales del niño para lograr cercanía, se piensa que disminuiría las expectativas de seguridad en la relación por parte del niño y llevaría al desarrollo de patrones de apego inseguros o desorganizados (Lecannelier, 2009). Por ende, la actitud desplegada por el cuidador tiene una influencia sustancial moderando el desarrollo de la capacidad para leer estados mentales propios y de otros (Brent, Holt, Keshavan, Seidman y Fonagy, 2014). En relación a los patrones de apego predominantes en esquizofrenia, Dozier et al. (2008) han señalado que el estilo desorganizado es el más prevalente. En un estudio con pacientes cursando un primer episodio psicótico, Macbeth, Gumley, Schwannauer y Fisher (2011) encontraron una mayor proporción de sujetos con patrón evitante utilizando la Adult Attachment Interview (AAI). Cabe destacar que este trabajo solo consideró el patrón seguro, inseguro/evitante e inseguro/preocupado, no incorporando el estilo desorganizado. El predominio del estilo evitante también ha sido reportado por otros autores (Harder y Daniel, 2014). Por su parte Bucci, Emsley y Berry (2017) estudiaron a 588 sujetos que cursaban psicosis no afectiva. Los clasificaron en apego seguro, ansioso, evitante y desorganizado utilizando la Psychosis Attachment Measure (PAM), encontrando mayor prevalencia del patrón seguro en la muestra. Como se puede apreciar estos trabajos utilizaron diferentes instrumentos de evaluación y muestran predominio de distintos patrones de apego. La necesidad de conocer con mayor profundidad la naturaleza de los déficits metacognitivos tiene implicancias clínicas y terapéuticas. Dentro de las primeras, los pacientes con Esquizofrenia al carecer de habilidades para comprender los estados mentales propios y ajenos, pueden presentar serias dificultades para asociar los hechos cotidianos con el pasado o prever un futuro que pudiera ser afectado por la acción presente. Por ende, estos déficits pueden llevar al individuo a una pérdida del sentido de identidad personal, una profunda desmoralización y pérdida global de significado. En este sentido, las personas con Esquizofrenia presentan dificultades no solo por deterioro cognitivo y síntomas, sino también por importantes alteraciones en la capacidad de interpretar y dar sentido a los desafíos de la vida cotidiana (Lysaker et al., 2016). En otras palabras, mientras los síntomas y alteraciones neurocognitivas hacen difícil ver cómo hacer ciertas cosas, los déficits metacognitivos limitarían la capacidad de poder ver el por qué deberían seguir ciertos cursos de acción. Estas alteraciones dejarían a los pacientes con una menor habilidad para construir los tipos de significado que sustentan conductas dirigidas a metas en contextos sociales, vocacionales y comunitarios. Desde una perspectiva terapéutica y rehabilitadora, en la actualidad el abordaje de la disfunción social y la sintomatología negativa a través del entrenamiento en habilidades sociales, intervenciones cognitivas y psicofarmacológicas, descuidan las necesidades que las personas tienen para entender las 
conexiones significativas que existen entre motivaciones, sentimientos, pensamientos y expectativas, repercutiendo en pobres resultados en el manejo de estas áreas (Lysaker y Dimaggio, 2014a). Similares observaciones se han realizado sobre apego y la importancia de su incorporación en las intervenciones en esta población. Griffiths y McLeod (2020) han hecho hincapié sobre las menores probabilidades de una recuperación exitosa de no considerar en el plan de tratamiento de estos sujetos el impacto de patrones de apego inseguro en la búsqueda de ayuda. En la revisión bibliográfica realizada se han encontrado fundamentalmente estudios internacionales y se trata de algunos grupos de trabajo específicos. No se han encontrado trabajos nacionales que hayan evaluado metacognición, sintomatología y apego en sujetos con un primer episodio de Esquizofrenia. De la búsqueda, solo un estudio evaluaba metacognición y sintomatología en pacientes con Esquizofrenia de nuestro país, pero se trata de pacientes portadores de psicosis de larga data (Lysaker et al., 2018). En sintonía con lo planteado por Lysaker et al. (2018), la mayoría de lo que conocemos sobre metacognición en Esquizofrenia se ha obtenido a partir de estudios realizados en muestras de pacientes angloparlantes de América del Norte. Considerando las diferencias culturales, históricas y lingüísticas, junto a su impacto potencial en cómo los pacientes pudieran integrar esa información sobre sí mismo y otros, es que resulta necesario replicar estudios sobre metacognición en otras culturas como la nuestra. En el presente trabajo se pretende conocer la capacidad metacognitiva y los patrones de apego preponderantes de un grupo de pacientes que cursan un primer episodio de Esquizofrenia y la relación de dichas capacidades metacognitivas con la sintomatología y patrones de apego en esta población. A la luz de la literatura revisada, se hipotetiza encontrar escasas habilidades metacognitivas, con predominio de patrones de apego inseguro (fundamentalmente rechazante), en que menores capacidades metacognitivas se vinculen a mayor sintomatología negativa, síntomas de desorganización y a patrones de apego inseguros.

\section{Método}

Se trata de un estudio cuantitativo, relacional, prospectivo, de corte transversal y observacional (Hernández, 2010). Se consideró la población de pacientes con diagnóstico confirmado de Esquizofrenia primer episodio del Servicio de Psiquiatría del Hospital Regional de Talca, de acuerdo a los criterios CIE-10, entre enero 2016 y diciembre 2018. Tanto pacientes como sus tutores dieron consentimiento voluntario para participar en el estudio. Éste contó con la aprobación del Comité de ética de la Universidad de Santiago de Chile y del Comité de ética científico del Servicio de Salud Maule. La información sobre los sujetos diagnosticados con Esquizofrenia se obtuvo de los registros de SISMAULE, sistema de registro 
estadístico del Servicio de Salud Maule, en que los diagnósticos se codifican según CIE-10. Además, se consultó a sus psiquiatras tratantes y fichas clínicas correspondientes para confirmar que los diagnósticos inscritos en el sistema fueran concordantes con el diagnóstico clínico actual. Este procedimiento fue necesario ya que muchos pacientes ingresados inicialmente como Esquizofrenia primer episodio, fueron registrados posteriormente como psicosis secundarias a consumo de sustancias o psicosis crónicas de larga data. Se consideró como criterio de exclusión el cursar fase aguda del trastorno de acuerdo a los parámetros establecidos en la Guía Clínica para el tratamiento del primer episodio en Esquizofrenia del Ministerio de Salud de Chile (Ministerio de Salud, 2009). Dos personas rechazaron participar en el estudio y sólo una cursaba fase aguda del trastorno, por lo que se contó finalmente con un total de 31 participantes con diagnóstico confirmado de Esquizofrenia primer episodio del Servicio de Psiquiatría del Hospital Regional de Talca.

\section{Instrumentos}

Los instrumentos fueron aplicados por el autor a cada participante en una sesión de dos horas, con diez minutos de descanso entre la aplicación de los instrumentos. Para evitar interferir en el desempeño cognitivo de los participantes, la sesión se inició aplicando la Indiana Psychiatric Illness Interview (IPII) que busca evocar narrativas, seguida de la Escala de Síndromes Positivo y Negativo (PANSS), finalizando con el Cartes: Modèles Individuels de Relation (CAMIR) cuestionario de carácter más lúdico.

Indiana Psychiatric Illness Interview (IPII). La IPII es una entrevista semiestructurada desarrollada para evaluar cómo las personas describen su narrativa de vida y comprenden su experiencia de padecer un trastorno de salud mental (Lysaker, Clements, Plascak-Hallberg, Knipscheer y Wright, 2002). Su aplicación demora alrededor de 30 minutos y se divide en cinco secciones: inicia con una narración general libre sobre la historia de su vida y prosigue con una narración sobre la experiencia de padecer un problema de salud mental. Posteriormente, se pregunta sobre qué ha sido y no ha sido afectado en su vida interpersonal y psicológica por la enfermedad. La cuarta sección trata sobre el grado de influencia del problema de salud mental en su vida. La entrevista finaliza preguntando si esperan permanecer igual y qué será diferente en el futuro. En este trabajo se utilizó la versión traducida al español por Leonor Irarrázaval (Lysaker et al., 2016).

Metacognitive Assessment Scale-Abbreviated (MAS-A). La MAS-A es una escala que evalúa las habilidades metacognitivas tal como se manifiestan en las narrativas de los participantes (Lysaker et al., 2005). La MAS-A está compuesta por cuatro subescalas y un puntaje total, cuyos valores reflejan jerárquicamente las habilidades metacognitivas logradas. En otras 
palabras, un mayor puntaje total o de una subescala se traduce en el despliegue de capacidades metacognitivas más complejas y avanzadas por parte del sujeto. Las subescalas corresponden a los dominios de la metacognición: Autorreflexividad (puntaje de 0-9), comprensión de la mente de otros (puntaje de 0-7), Descentración (puntaje de 0-3) y Maestría (puntaje de 0-9). La suma de ellas representa la capacidad metacognitiva total (puntaje de 028). Las subescalas son revisadas individualmente tomando los datos de las transcripciones obtenidas de la IPII y posteriormente puntuadas por cada función que el evaluador considera como lograda. Si la función es lograda completamente se otorga 1 punto, parcialmente lograda 0,5 punto y si no se cumple 0 punto. En este estudio se aplicó la versión traducida al español por Leonor Irarrázaval (Lysaker et al., 2016). Los datos obtenidos con la versión norteamericana de la MAS-A reportan valores de consistencia interna y fiabilidad test-retest e inter-jueces aceptables, con coeficientes intraclase entre 0,71 y 0,91 (Lysaker et al, 2005; Lysaker y Salyers, 2007).

Cartes: Modèles Individuels de Relation (CAMIR). CAMIR es un cuestionario basado en la teoría del apego, de auto-reporte, que evalúa los modelos operantes internos en adultos y otras escalas relacionadas al apego. Se utilizó la versión validada para población chilena de Garrido, Santelices, Pierrehubert y Armijo (2009) que consta de un formato de respuesta tipo Likert de 1 a 5 y cuenta con 71 ítems. Dispone de tres prototipos de Q-Sorts: Seguro, Rechazante y Preocupado, entregando puntajes en 13 factores de variables relacionadas a los patrones de apego y para cada uno de los prototipos de apego, describiendo el caso predominante (Garrido et al., 2009). Estos 13 factores de variables (escalas de A a M) son: Escala A: Interferencia Parental (IP); Escala B: Preocupación Familiar (PF); Escala C: Resentimiento de Infantilización (RI); Escala D: Apoyo Parental (AP); Escala E: Apoyo Familiar (AF); Escala F: Reconocimiento de Apoyo (RA); Escala G: Indisponibilidad Parental (IP2); Escala H: Distancia Familiar (DF); Escala I: Resentimiento de Rechazo (RR); Escala J: Traumatismo Parental (TP); Escala K: Bloqueo de Recuerdos (BR); Escala L: Dimisión Parental (DP); Escala M: Valorización de Jerarquía (VJ). Con el fin de obtener el estilo de apego predominante de cada sujeto, se combinan los puntajes escalares correspondientes a cada uno de los patrones de apego obteniéndose un perfil para cada persona. Un puntaje sobre 60 en cualquier escala marca una tendencia a que el estilo de apego sea ése. Además, al margen de estos tres prototipos el cuestionario puede entregar un estilo de apego Inclasificable (anteriormente considerado Desorganizado). Este cuarto patrón se considera cuando existe más de una escala dominante con puntaje mayor de 60 puntos. La validación de CAMIR en Chile encontró una fiabilidad adecuada, con valores alfa entre 0.72 y 0.84 , salvo en las escalas IP ( $a=0.54)$, DF ( $a=0.33)$, DP ( $a=0.39)$ y VJ ( $a=0.33)$ (Garrido et al., 2009). 
Escala de Síndromes Positivo y Negativo (PANSS). La PANSS se trata de una entrevista y escala de observación cuyo objetivo es el de evaluar el Síndrome Esquizofrénico desde el punto de vista dimensional y categorial. Originalmente desarrollada por Kay, Fiszbein y Opler (1987) y adaptada al español por Peralta y Cuesta (1994), consta de 30 ítems, con formato de respuesta de tipo Likert de 1 a 7 . En el caso del presente estudio, se utilizó una variación de la versión original que toma los mismos ítems, pero los organiza en un modelo de cinco factores que permite valorar otras subescalas o síndromes: Positiva (puntaje de 7 a 49), Negativa (puntaje de 8-56), Desorganización (puntaje de 10 a 70), Excitación (puntaje de 8 a 56) y Distrés emocional (puntaje de 8 a 56). Mayores puntajes en cada subescala indicarían mayor gravedad de la sintomatología para el síndrome correspondiente (Van der Gaag et al., 2006). Este modelo de 5 factores permite valorar otros síndromes (Desorganización, Excitación y Distrés emocional) que la escala original no permite y que resultan de interés para este estudio. Los valores de consistencia interna del modelo de cinco factores de la PANSS fueron a Positiva $=0.73 ;$ a Negativa $=0.83 ;$ a Desorganización $=0.81 ; a$ Excitación $=0.70 ; a$ Distrés emocional= 0.64 (Bagaric, Brecic, Ostojic, Jukic y Goles, 2013).

\section{Análisis de datos}

Se tomó la información recolectada a través de los instrumentos señalados y se estructuró una base de datos desarrollada para este proyecto. Para el análisis de datos se utilizó el programa estadístico SPSS versión 21 para Mac. Se llevó a cabo un análisis estadístico descriptivo, con distribución de frecuencias, medidas de tendencia central y medidas de variabilidad para los datos recogidos de las variables en estudio. En cuanto al análisis inferencial, se evaluó la relación entre las variables cuantitativas metacognición y sintomatología utilizando el coeficiente de correlación no paramétrico de Spearman. Se realizó análisis de varianza para verificar diferencias significativas de metacognición según los patrones de apego. Se verificaron los supuestos de normalidad mediante el test de Shapiro Wilk y homogeneidad de varianzas mediante el test $\mathrm{F}$ de Levene de los residuos para metacognición (MAS-A) y sintomatología (PANSS) según patrón de apego, cumpliéndose el supuesto de normalidad. Para valorar si existieron diferencias estadísticamente significativas en las capacidades metacognitivas y sintomatología para los cuatro estilos de apego de CAMIR se aplicó el test F de Anova. Por otra parte, se utilizó la prueba t de Student para evaluar la presencia de diferencias estadísticamente significativas de las capacidades metacognitivas y sintomatología al segmentar la población en patrón de apego seguro e inseguro. No hubo datos perdidos. El nivel de significancia se estableció en 0.05. 


\section{Resultados}

El análisis descriptivo para las variables demográficas, capacidades metacognitivas, patrones de apego y sintomatología se resume en la Tabla 1. La media de edad de la muestra fue 22 años, la mayoría de los participantes son de sexo masculino $(77,4 \%)$ y la totalidad de la muestra es soltero(a). Cerca de la mitad $(45,2 \%)$ no completó la enseñanza media y ninguno terminó los estudios superiores. En cuanto al tratamiento, el $71 \%$ solo usa antipsicóticos de segunda generación, el $12,9 \%$ la asociación de antipsicóticos de primera y segunda generación y este mismo porcentaje no utiliza medicación antipsicótica. La media del puntaje total de la MAS-A de la muestra fue de 11,2, de la escala Autorreflexibidad 4,1, Comprensión de la Mente de otros 3,0, Descentración 0,8 y Maestría 3,3. Sobre la categorización del patrón de apego, el tipo más prevalente resultó ser el Rechazante (evitante) (51,6\%), seguido del tipo Seguro $(22,6 \%$ ) y un $12,9 \%$ tanto para el tipo Preocupado (ansioso ambivalente) como para el Inclasificable (desorganizado). El patrón inseguro dio cuenta del $77,4 \%$ de la muestra. La media de los puntajes sobre sintomatología psicótica en la PANSS, considerando los 5 factores propuestos por Van der Gaag (2006), fue de 15,8 en la subescala Positiva, 20,5 en la Negativa, 25,3 en la de Desorganización, 17,1 en la de Excitación y 19,8 en la de Distrés Emocional. 
Tabla 1 : Variables demográficas, puntajes metacognitivos, patrones de apego y sintomatología en Esquizofrenia primer episodio en HRT.

\begin{tabular}{|c|c|c|c|c|c|c|}
\hline & Frecuencia & $\begin{array}{c}\text { Porcentaje } \\
(\%)\end{array}$ & Media & Moda & Mediana & $\begin{array}{l}\text { Desviación } \\
\text { estándar }\end{array}$ \\
\hline Edad & & & 22,0 & 25 & 22,0 & 5,7 \\
\hline \multicolumn{7}{|l|}{ Sexo } \\
\hline Masculino & 24 & 77,4 & & & & \\
\hline Femenino & 7 & 22,6 & & & & \\
\hline \multicolumn{7}{|l|}{ Escolaridad } \\
\hline Básica incompleta & 1 & 3,2 & & & & \\
\hline Básica completa & 2 & 6,5 & & & & \\
\hline Media incompleta & 11 & 35,6 & & & & \\
\hline Media completa & 8 & 25,8 & & & & \\
\hline Superior incompleta & 9 & 29,0 & & & & \\
\hline Superior completa & 0 & 0 & & & & \\
\hline \multicolumn{7}{|l|}{ Uso de antipsicóticos } \\
\hline Sin antipsicóticos & 4 & 12,9 & & & & \\
\hline $1^{\circ}$ generación & 1 & 3,2 & & & & \\
\hline $2^{\circ}$ generación & 22 & 71,0 & & & & \\
\hline $1^{\circ}$ y $2^{\circ}$ generación & 4 & 12,9 & & & & \\
\hline \multicolumn{7}{|l|}{ Puntaje MAS-A } \\
\hline Autorreflexividad & & & 4,11 & 3,5 & 4,0 & 1,09 \\
\hline Comprensión mente otros & & & 3,03 & 2,5 & 3,0 & 0,95 \\
\hline Descentración & & & 0,82 & 1,0 & 1,0 & 0,62 \\
\hline Maestría & & & 3,32 & 3,0 & 3,0 & 1,61 \\
\hline MAS-A total & & & 11,29 & 8,5 & 10,5 & 3,74 \\
\hline \multicolumn{7}{|l|}{ Apego } \\
\hline Seguro & 7 & 22,6 & & & & \\
\hline Rechazante & 16 & 51,6 & & & & \\
\hline Preocupado & 4 & 12,9 & & & & \\
\hline Inclasificable/Desorganizado & 4 & 12,9 & & & & \\
\hline \multicolumn{7}{|l|}{ PANSS } \\
\hline Positiva & & & 15,87 & 15 & 16,0 & 5,34 \\
\hline Negativa & & & 20,52 & 14 & 20,0 & 8,11 \\
\hline Desorganización & & & 25,35 & 27 & 26,0 & 6,63 \\
\hline Excitación & & & 17,16 & 14 & 15,0 & 4,64 \\
\hline Distrés emocional & & & 19,87 & 19 & 19,0 & 4,93 \\
\hline
\end{tabular}

Nota: MAS-A: Metacognition Assessment Scale Abbreviated. PANSS: Positive and Negative Syndrome Scale. 
Las asociaciones entre metacognición y sintomatología se resumen en la Tabla 2. El puntaje total de la MAS-A se asoció significativamente con todas las subescalas de la PANSS, a excepción de la subescala Excitación. Presentó correlación negativa significativa con la subescala Desorganización de la PANSS ( $r h o=-0,861, p<0,0001$ ), la subescala Negativa (rho= $-0,761, p<0,0001$ ), la subescala Positiva ( $r h o=-0,430, p=0,016$ ) y la subescala Distrés emocional (rho= $-0,366, p=0,043$ ). El dominio Autorreflexividad obtuvo relación negativa significativa con las subescalas Desorganización ( $r$ o $=-0,777, p<0,0001$ ) y Negativa ( $r$ o $=-0,612, p<0,0001$ ). Comprensión de la mente de otros se relacionó significativamente con la subescala Desorganización ( $r h o=-0,642, p<0,0001$ ), Negativa ( $r h o=-0,698, p<0,0001$ ) y Distrés emocional (rho= $-0,359, p=0,048$ ). Descentración presentó relación negativa significativa con todas las subescalas de la PANSS, siendo mayor con la subescala Desorganización. La habilidad Maestría se relacionó significativamente con la subescala Desorganización (rho= -0,759, $\mathrm{p}<$ 0,0001), Negativa (rho $=-0,698, p<0,0001$ ) y Positiva (rho= -0,466, $p=0,008$ ). No hubo correlación estadísticamente significativa con Excitación y Distrés emocional.

Tabla 2 : Correlaciones entre metacognición y sintomatología en Esquizofrenia primer episodio en HRT.

\begin{tabular}{|c|c|c|c|c|c|}
\hline & $\begin{array}{c}\text { MAS-A } \\
\text { Total }\end{array}$ & $\begin{array}{c}\text { MAS-A } \\
\text { Autorreflexividad }\end{array}$ & $\begin{array}{c}\text { MAS-A } \\
\text { Comprensión de } \\
\text { la mente de } \\
\text { otros }\end{array}$ & $\begin{array}{c}\text { MAS-A } \\
\text { Descentración }\end{array}$ & $\begin{array}{l}\text { MAS-A } \\
\text { Maestría }\end{array}$ \\
\hline PANSS &,$- 430 *$ &,- 252 &,- 306 &,$- 473 * *$ &,$- 466 * *$ \\
\hline \multicolumn{6}{|l|}{ Positiva } \\
\hline PANSS Negativa &,$- 761^{* *}$ &,$- 612^{* *}$ &,$- 698^{* *}$ &,$- 558^{* *}$ &,$- 698^{* *}$ \\
\hline PANSS &,$- 861^{* *}$ &,- 777 ** &,$- 642^{* *}$ &,$- 654^{* *}$ &,$- 759 * *$ \\
\hline \multicolumn{6}{|l|}{ Desorganización } \\
\hline PANSS Excitación & -.321 &,- 203 &,- 278 &,$- 414^{*}$ &,- 281 \\
\hline $\begin{array}{l}\text { PANSS Distrés } \\
\text { emocional }\end{array}$ &,$- 366^{*}$ &,- 342 &,$- 359^{*}$ &,$- 446^{*}$ &,- 326 \\
\hline
\end{tabular}

Nota: Correlación de Spearman, rho. MAS-A: Metacognition Assessment Scale Abbreviated. PANSS: Positive and Negative Syndrome Scale. * $p £ 0,05$, la correlación es significativa al nivel 0,05 (bilateral)

** $p £ 0,01$, la correlación es significativa al nivel 0,01 (bilateral) 
Las capacidades metacognitivas según los distintos patrones de apego se enlistan en la Tabla 3. El promedio de la puntuación de la MAS-A total y de sus dominios resultó ser menor en el patrón de apego inseguro en comparación al seguro, a excepción del dominio Descentración. Al comparar la media de los dominios metacognitivos según apego Seguro, Rechazante, Preocupado o Inclasificable, los puntajes más bajos se obtuvieron en el patrón Inclasificable/Desorganizado para MAS-A total, Autorreflexividad, Comprensión de la mente de otros y Descentración, seguido del estilo Preocupado.

Tabla 3 : Puntajes metacognitivos de la MAS-A y sintomatología de la PANSS en Esquizofrenia primer episodio según patrón de apego.

\begin{tabular}{|c|c|c|c|c|}
\hline & $\begin{array}{c}\text { Apego Seguro. } \\
\text { Media (DS) }\end{array}$ & $\begin{array}{c}\text { Apego } \\
\text { Rechazante. } \\
\text { Media (DS) }\end{array}$ & $\begin{array}{c}\text { Apego } \\
\text { Preocupado. } \\
\text { Media (DS) }\end{array}$ & $\begin{array}{c}\text { Apego } \\
\text { Inclasificable/ } \\
\text { Desorganizado. } \\
\text { Media (DS) }\end{array}$ \\
\hline MAS-A total & $\begin{array}{c}11,50 \\
(4,28)\end{array}$ & $\begin{array}{c}11,78 \\
(3,82)\end{array}$ & $\begin{array}{l}10,62 \\
(4,40)\end{array}$ & $\begin{array}{c}9,62 \\
(2,28)\end{array}$ \\
\hline MAS-A Autoreflexividad & $\begin{array}{l}4,21 \\
(1,18)\end{array}$ & $\begin{array}{l}4,28 \\
(1,11)\end{array}$ & $\begin{array}{l}3,87 \\
(1,43)\end{array}$ & $\begin{array}{c}3,50 \\
(0,40)\end{array}$ \\
\hline $\begin{array}{l}\text { MAS-A Comprensión de la } \\
\text { mente de otros }\end{array}$ & $\begin{array}{l}3,14 \\
(1,21)\end{array}$ & $\begin{array}{l}3,00 \\
(0,83)\end{array}$ & $\begin{array}{c}3,12 \\
(1,43)\end{array}$ & $\begin{array}{c}2,87 \\
(0,75)\end{array}$ \\
\hline MAS-A Descentración & $\begin{array}{c}0,78 \\
(0,63)\end{array}$ & $\begin{array}{l}0,96 \\
(0,61)\end{array}$ & $\begin{array}{c}0,87 \\
(0,62)\end{array}$ & $\begin{array}{l}0,25 \\
(0,5)\end{array}$ \\
\hline MAS-A Maestría & $\begin{array}{l}3,35 \\
(1,77)\end{array}$ & $\begin{array}{l}3,53 \\
(1,74)\end{array}$ & $\begin{array}{r}2,75 \\
(1,25)\end{array}$ & $\begin{array}{l}3,00 \\
(1,47)\end{array}$ \\
\hline PANSS Positiva & $\begin{array}{l}16,86 \\
(7,60)\end{array}$ & $\begin{array}{l}14,56 \\
(4,53)\end{array}$ & $\begin{array}{l}16,50 \\
(5,26)\end{array}$ & $\begin{array}{l}18,75 \\
(4,11)\end{array}$ \\
\hline PANSS Negativa & $\begin{array}{l}19,29 \\
(6,44)\end{array}$ & $\begin{array}{l}19,06 \\
(6,48)\end{array}$ & $\begin{array}{l}23,50 \\
(9,32)\end{array}$ & $\begin{array}{l}28,00 \\
(2,94)\end{array}$ \\
\hline PANSS Desorganización & $\begin{array}{l}24,43 \\
(8,46)\end{array}$ & $\begin{array}{l}24,31 \\
(5,85)\end{array}$ & $\begin{array}{l}28,50 \\
(9,32)\end{array}$ & $\begin{array}{l}28,00 \\
(2,94)\end{array}$ \\
\hline PANSS Excitación & $\begin{array}{l}18,29 \\
(5,49)\end{array}$ & $\begin{array}{l}16,25 \\
(3,78)\end{array}$ & $\begin{array}{l}18,25 \\
(7,84)\end{array}$ & $\begin{array}{l}17,75 \\
(3,50)\end{array}$ \\
\hline PANSS Distrés emocional & $\begin{array}{l}20,43 \\
(5,15)\end{array}$ & $\begin{array}{l}18,69 \\
(4,23)\end{array}$ & $\begin{array}{l}18,25 \\
(6,18)\end{array}$ & $\begin{array}{l}25,25 \\
(3,40)\end{array}$ \\
\hline
\end{tabular}

Nota: Los datos se enlistan como: media (desviación estándar). MAS-A: Metacognition Assessment Scale Abbreviated. PANSS: Positive and Negative Syndrome Scale. 
No se encontraron diferencias estadísticamente significativas en las medias de los puntajes de la capacidad metacognitiva total para los cuatro patrones de apego al aplicar el test $F$ de Anova (valor $p=0,768>0,05$ ). Por otra parte, no existieron diferencias estadísticamente significativas en la media de la MAS-A total al aplicar t de Student (valor $p=0,870>0,05$ ) cuando se segmentó la población en apego seguro e inseguro. Tampoco se encontraron diferencias estadísticamente significativas al considerar la media de los distintos dominios metacognitivos de la MAS-A, tanto para los cuatro patrones de apego descritos como para apego seguro e inseguro, lo cual se muestra en la Tabla 4. La media de los puntajes obtenidos de la PANSS para los cuatro patrones de apego se resume en la Tabla 3. No se encontraron diferencias estadísticamente significativas $(p>0,05)$ en las medias de los puntajes de las subescalas de la PANSS para los cuatro patrones de apego al aplicar el test F de Anova. Tampoco se encontraron diferencias estadísticamente significativas en las medias de los puntajes de las subescalas de la PANSS al aplicar t de Student tras segmentar la población en apego seguro e inseguro, como se aprecia en la Tabla 4.

Tabla 4 : Metacognición y sintomatología en Esquizofrenia primer episodio según los cuatro patrones de apego $(F)$ y tras segmentar en apego seguro e inseguro $(t)$.

\begin{tabular}{lcccc}
\hline & F calculado & $p$ & t de Student & $p$ \\
& & & \\
& & & & \\
MAS-A total & 0,38 & 0,76 & 0,16 & 0,87 \\
MAS-A Autoreflexividad. & 0,60 & 0,62 & 0,27 & 0,78 \\
MAS-A Comprensión de & 0,08 & 0,92 & 0,34 & 0,73 \\
la mente de otros. & & & $-0,17$ & 0,86 \\
MAS-A Descentración. & 1,49 & 0,24 & 0,06 & 0,95 \\
MAS-A Maestría & 0,29 & 0,83 & 0,55 & 0,59 \\
PANSS Positiva & 0,78 & 0,51 & $-0,45$ & 0,66 \\
PANSS Negativa & 0,89 & 0,45 & $-0,41$ & 0,68 \\
PANSS Desorganización & 0,66 & 0,58 & 0,72 & 0,48 \\
PANSS Excitación & 0,41 & 0,74 & 0,34 & 0,74 \\
PANSS Distrés emocional & 2,34 & 0,09 & & \\
\hline
\end{tabular}

Nota: MAS-A: Metacognition Assessment Scale Abbreviated. PANSS: Positive and Negative Syndrome Scale. 


\section{Discusión}

Uno de los objetivos de este estudio, consistía en evaluar la capacidad metacognitiva de los pacientes diagnosticados con Esquizofrenia primer episodio en el Servicio de Psiquiatría del Hospital Regional de Talca, entre los años 2016-2018. La baja capacidad metacognitiva obtenida de los puntajes de la MAS-A total y sus dominios, son concordantes con los resultados en otras muestras con primer episodio psicótico (Trauelsen et al., 2016) y de pacientes con Esquizofrenia de larga data en nuestro país (Lysaker et al., 2018). Por otra parte, los puntajes son menores en relación a pacientes con diagnóstico de Trastorno Bipolar (Tas et al., 2014, Lysaker et al., 2018), Trastorno por uso de sustancias (Vohs et al., 2014) y grupos control no clínicos (Lysaker et al., 2018). Los resultados respaldan los hallazgos de aquellos estudios en que sujetos diagnosticados con Esquizofrenia primer episodio tendrían menores capacidades metacognitivas en comparación a población no afectada por esta enfermedad. En este trabajo, la media de los puntajes de Autorreflexividad en los sujetos participantes nos señala que logran identificar operaciones cognitivas y emociones propias, sin lograr subfunciones de Diferenciación ni de Integración de los propios estados mentales. Además, solo logran identificar operaciones cognitivas ajenas con grave dificultad para adoptar una postura no egocentrista sobre los acontecimientos que les rodean. Sobre el dominio Maestría, la media de las puntuaciones de los participantes nos muestra que consiguen definir un problema psicológico o interpersonal plausible, ante el cual solo logran desplegar estrategias pasivas o de primer nivel para abordarlos. En cuanto al patrón de apego más frecuente en este estudio, resultó ser el tipo Rechazante $(51,6 \%)$, seguido por el tipo Seguro y por último el tipo Preocupado y Desorganizado. Este hallazgo es concordante con los resultados reportados por MacBeth et al. (2011) quienes evaluaron apego en una muestra de primer episodio psicótico a través de la Adult Attachment Interview, obteniendo predominancia del tipo Rechazante $(61,8 \%)$, seguido del tipo Seguro y finalmente el Preocupado. Conceptualmente, la teoría del apego se ha considerado útil como medio para entender las bases interpersonales y del desarrollo del proceso de recuperación y adaptación en psicosis (Gumley et al., 2014), en que las conductas de apego inseguros serían una estrategia implícita para lidiar con afectos potencialmente desestabilizadores (MacBeth et al., 2011). El hecho de encontrar alta frecuencia de apego inseguro debe alertar considerando los hallazgos de Dozier (como se citó en Gumley y Schwannauer, 2008), en que estos sujetos eran menos propensos a comentar de su sintomatología a otros, tendían a minimizar la intervención de los profesionales de salud y tenían menor probabilidad de adherir a tratamiento. 
Sobre el análisis de la correlación entre metacognición y sintomatología, las asociaciones más fuertes se dieron entre la capacidad metacognitiva total y sus dominios, con las subescalas Desorganización y Negativa de la PANSS. Estos resultados concuerdan con los hallazgos de estudios internacionales previos sobre la asociación de baja capacidad metacognitiva con sintomatología negativa y de desorganización en pacientes angloparlantes con primer episodio psicótico (Hamm et al., 2012, MacBeth et al., 2014, Vohs et al., 2014, MacBeth et al., 2015, Trauelsen et al., 2016) y permite plantear la hipótesis de que se podrían encontrar déficits metacognitivos a la base de las disrupciones en la organización del pensamiento y la sintomatología negativa en sujetos que padecen Esquizofrenia, ya desde un primer episodio (Lysaker et al., 2015). Esta hipótesis también ha sido compartida por otros autores, quienes han propuesto que la dificultad inicial de acceder a los estados mentales propios y ajenos en el contexto de interacciones sociales, llevaría a procesos de retraimiento social exacerbando las dificultades interpersonales que presentan. En este tipo de situaciones los pacientes presentarían importantes dificultades para poder identificar operaciones cognitivas y emocionales de otros, aumentando la confusión e hiperarousal en ellos, lo cual exacerbaría la evitación de situaciones sociales repercutiendo en mayores déficits metacognitivos a futuro (Salvatore, Dimaggio y Lysaker, 2007, MacBeth et al., 2014). Teniendo en consideración estas observaciones, los resultados obtenidos en sujetos con primer episodio llevan a plantearnos la duda sobre la presencia de déficits metacognitivos en etapas premórbidas de Esquizofrenia, tal como lo han señalado Vohs et al. (2014). En relación a los otros dominios sintomáticos, al analizar la falta de correlación estadísticamente significativa de metacognición con la subescala Excitación de la PANSS, compartimos las observaciones realizadas por MacBeth et al. (2015) al señalar que su escasa asociación se podría explicar por el hecho de que este síndrome es representado por alteraciones predominantemente conductuales y psicomotoras, a diferencia de lo que metacognición esencialmente representa. En cuanto al análisis de la asociación de metacognición y apego, la presencia de una menor capacidad metacognitiva en apego inseguro, principalmente en el patrón Inclasificable/Desorganizado, puede ser comprendido según el concepto de Modelos Múltiples de Bowlby. Este concepto se trata de la presencia de información incompatible que no puede ser cierta a la vez del mismo aspecto de la realidad, en que aparecen múltiples modelos que abordan la experiencia cuando debiera ser solo uno y sin contradicción. Estas situaciones serían prototípicas de patrones de apego más desfavorables, por ende, estos modelos múltiples se podrían apreciar en dichos contextos. Así, el monitoreo metacognitivo será más dificultoso ante la presencia de estos modelos con información distorsionada y desorganizada en el sujeto (Main, 1991). En nuestro trabajo no se encontraron diferencias estadísticamente significativas en las medias de metacognición total y sus subescalas según patrón de apego. El que no existan estas 
diferencias podría explicarse por el hecho de que metacognición ha sido considerada una capacidad que no se ve alterada exclusivamente por la activación del sistema de apego, sino que puede verse afectada también por disfunciones a nivel neurobiológico y otros sistemas motivacionales, por lo que existirían además otros factores que determinarían estas alteraciones metacognitivas (Dimaggio y Lysaker, 2015). Sobre las medias de los distintos síndromes de la PANSS para cada patrón de apego, este estudio no encontró diferencias estadísticamente significativas. Estos resultados concuerdan con los obtenidos por MacBeth et al. (2011) en población afectada por un primer episodio psicótico, quienes tampoco encontraron diferencias en la gravedad de los síndromes según estilo de apego. Por lo tanto, en el caso de los pacientes con Esquizofrenia primer episodio del HRT, la magnitud de la sintomatología psicótica no estaría influida por el patrón de apego de los sujetos afectados.

En un esfuerzo por integrar los resultados obtenidos en este trabajo, concordamos con la propuesta de Gumley y Liotti (2019), quienes han señalado que la aparición de sintomatología negativa y de desorganización en Esquizofrenia en el contexto de déficits metacognitivos graves, podrían ser entendidos como el resultado de intentos por desactivar el sistema de apego (como ocurre en el patrón de apego evitante) con el fin de poder lidiar con la desorganización y el miedo dentro de los vínculos significativos. Además, proponen que las alteraciones metacognitivas podrían darse como rasgo más estable vinculado a alteraciones neurocognitivas profundas y también darse como estado relacionado al distrés que activa el sistema de apego en determinadas situaciones (Gumley y Liotti, 2019, Harder y Daniel, 2014).

Las implicancias terapéuticas de estos hallazgos son relevantes. Se podría señalar que el desarrollo de intervenciones psicoterapéuticas que promuevan habilidades metacognitivas en sujetos diagnosticados con Esquizofrenia, incluso tempranamente desde un primer episodio, generarían cambios en la gravedad de los síntomas negativos y de desorganización, áreas donde la psicofarmacología no ha logrado tener los resultados que se esperan. Además, teniendo en cuenta que los síntomas negativos y patrones de apego inseguros pueden afectar la búsqueda de ayuda profesional determinando tratamientos menos efectivos, incorporar estas variables en el desarrollo y planificación de futuras intervenciones psicoterapéuticas con enfoque rehabilitador en esta población resultaría indispensable (MacBeth et al., 2014, MacBeth et al., 2015, Trauelsen et al., 2016, Vergara, 2018). Dentro de los aspectos metodológicos de este estudio, el uso de la PANSS y sus 5 factores propuesto por Van der Gaag (2006) fue un acierto en contraposición a las tres subescalas que utiliza la PANSS original. El uso del modelo de 5 factores permitió evidenciar la fuerte correlación negativa (estadísticamente significativa) entre síntomas de desorganización y metacognición. El uso del modelo original con las subescalas Positiva, Negativa y Psicopatología general, no habría 
permitido evidenciar dichos hallazgos. Por otra parte, este trabajo presenta limitaciones que resulta importante explicitar. Primero, se trata de un estudio correlacional por lo que permite conocer la presencia de asociaciones, pero no se pueden estimar relaciones causales entre las variables. Además, se ha utilizado el instrumento MAS-A que no está validado en nuestra población, por lo que para futuros trabajos es necesario que sea validado en nuestro país. Otro punto relevante, lo constituye la escasa cantidad de participantes con primer episodio de Esquizofrenia incluidos en el trabajo. Considerando la dificultad para reclutar un número mayor de pacientes, dado lo específico de las características de esta población, se podrían llevar a cabo futuros estudios multicéntricos, en colaboración con otros servicios clínicos del país.

\section{Referencias}

Bagaric, D., Brecic, P., Ostojic, D., Jukic, V., y Goles, A. (2013). The relationship between depressive syndrome and suicidal risk in patients with acute schizophrenia. Croatian Medical Journal, 54(5), 436-443. https://doi.org/10.3325/cmj.2013.54.436

Bleuler, E. (2011). Dementia praecox o el grupo de las esquizofrenias. Buenos Aires, Argentina: Polemos.

Brent, B. K., Holt, D. J., Keshavan, M. S., Seidman, L. J., y Fonagy, P. (2014). Mentalizationbased treatment for psychosis: linking an attachment-based model to the psychotherapy for impaired mental state understanding in people with psychotic disorders. The Israel Journal of Psychiatry and Related Sciences, 51(1), 17-24. https://tinyurl.com/ybrmfljd

Bucci, S., Emsley, R., y Berry, K. (2017). Attachment in psychosis: A latent profile analysis of attachment styles and association with symptoms in a large psychosis cohort. Psychiatry Research, 247, 243-249. https://doi.org/10.1016/j.psychres.2016.11.036

Buck, K.D., Warman, D.M., Huddy, V., y Lysaker, P.H. (2012). The relationship of metacognition with jumping to conclusions among persons with Schizophrenia spectrum disorders. Psychopathology, 45(5), 271-275. https://doi.org/10.1159/000330892

Davis, L. W., Eicher, A. C., y Lysaker, P. H. (2011). Metacognition as a predictor of therapeutic alliance over 26 weeks of psychotherapy in Schizophrenia. Schizophrenia Research, 129(1), 85-90. https://doi.org/10.1016/j.schres.2011.02.026.

Dimaggio, G., y Lysaker, P. H. (2015). Metacognition and Mentalizing in the psychotherapy of patients with psychosis and personality disorders. Journal of Clinical Psychology, 71(2), 117124. https;//doi.org/10.1002/jclp.22147 
Dozier, M., Stovall-McClough, C., y Albus, K. E. (2008). Attachment and psychopatology in adulthood. En J. Cassidy y P. Shaver (Eds.). Handbook of attachment. Theory, research, and clinical applications. (pp. 718-744). New York, USA: Guilford Press.

Flavell, J. H. (1979). Metacognition and cognitive monitoring. A new area of cognitivedevelopmental inquiry. American Psychologist, 34(10), 906-911. https://doi.org/10.1037/0003-066x.34.10.906.

Garrido, L., Santelices, M. P., Pierrehumbert, B., y Armijo, I. (2009). Validación chilena del cuestionario de evaluación de apego en el adulto CAMIR. Revista Latinoamericana de Psicologia, 41(1), 81-98.

Griffiths, H., y McLeod, H. (2020). Promoting recovery from negative symptoms. An attachment theory perspective. En K. Berry, S. Bucci y A. Danquah (Eds.). Attachment Theory and Psychosis. (pp. 62-83). London, UK: Routledge.

Gumley, A., y Schwannauer, M. (2008). Volver a la normalidad después de un Trastorno Psicótico. Un modelo cognitivo-relacional para la recuperación y la prevención de recaídas. Bilbao, España: Editorial Desclée de Brouwer.

Gumley, A. I., Schwannauer, M., MacBeth, A., Fisher, R., Clark, S., Rattrie, L., y Birchwood, M. (2014). Insight, duration of untreated psychosis and attachment in first-episode psychosis: Prospective study of psychiatric recovery over 12-month follow-up. British Journal of Psychiatry, 205(1), 60-67. https://doi.org/10.1192/bjp.bp.113.126722P

Gumley, A., y Liotti, G. (2019). An Attachment perspective on Schizophrenia: The role of disorganized attachment, dissociation, and mentalization. En A. Moskowitz, M. Dorahy y I. Schäfer (Eds.). Psychosis, Trauma and Dissociation. (pp. 97-116). Hoboken, NJ, USA: John Wiley \& Sons.

Hamm, J.A., Renard, S.B., Fogley, R. L., Leonhardt, B.L., Dimaggio, G., Buck, K.D., y Lysaker, P. H. (2012). Metacognition and Social Cognition in Schizophrenia: Stability and relationship to current and prospective symptom assessment. Journal of Clinical Psychology, 68(12), 13031312. https://doi.org/10.1002/jclp. 21906

Harder, S., y Daniel, S. (2014). The relationship between metacognitive profile, attachment pattern, and intersubjective process in psychotherapy of a person recovering from firstepisode schizophrenia. En P.H Lysaker, G. Dimaggio y M. Brüne (Eds.). Social Cognition and Metacognition in Schizophrenia. Psychopathology and Treatment Approaches. (pp. 261283). London, UK: Academic Press

Hernández, R. (2010). Metodología de la Investigación. Mexico. D.F, Mexico: McGraw-Hill. 
Kay, S. R., Fiszbein, A., y Opler, L. A. (1987). The Positive and negative syndrome scale (PANSS) for Schizophrenia. Schizophrenia Bulletin, 13(2), 261-276. https://doi. org/10.1093/schbul/13.2.261

Lecannelier, F. (2012). Conocimiento \& complejidad: Una perspectiva evolucionista. Santiago, Chile: LOM Ediciones.

Lecannelier, F. (2009). Apego e Intersubjetividad. Segunda parte: la teoría del apego. Santiago, Chile: LOM Ediciones.

Lysaker P. H., Clements C. A., Plascak-Hallberg C. D., Knipscheer S. J., y Wright D. E. (2002). Insight and Personal Narrative of Illness in Schizophrenia. Psychiatry, 65(3), 197-206. https://doi.org/10.1521/psyc.65.3.197.20174

Lysaker, P. H., Carcione, A., Dimaggio, G., Johannesenn, J. K., Nicolò, G., Procacci, M., y Semerari, A. (2005). Metacognition amidst narratives of self and illness in Schizophrenia: Associations with neurocognition, symptoms, insight and quality of life. Acta Psychiatrica Scandinavica, 112(1), 64-71. https://doi.org/10.1111/j.1600-0447.2005.00514.x

Lysaker, P. H., y Salyers, M. P. (2007). Anxiety symptoms in schizophrenia spectrum disorders: Associations with social function, positive and negative symptoms, hope and trauma history. Acta Psychiatrica Scandinavica, 116(4), 290-298. https://doi.org/10.1111/j.16000447.2007.01067.x.

Lysaker, P. H., Dimaggio, G., Carcione, A., Procacci, M., Buck, K. D., Davies, L. W. ,... y Nicolò, G. (2010a). Metacognition and schizophrenia: the capacity for self -reflexivity as a predictor for prospective assessment of work performance over six months. Schizophrenia Research, 122(1-3), 124-130. https://doi.org/10.1016/j.schres.2009.04.024

Lysaker, P. H., Shea, A. M., Buck, K. D., Dimaggio, G., Nicolò, G., Procacci, M., ... y Rand, K. L. (2010b). Metacognition as a mediator of the effects of impairments in neurocognition on social function in Schizophrenia spectrum disorders. Acta Psychiatrica Scandinavica, 122(5), 405-413. https://doi.org/10.1111/j.1600-0447.2010.01554.x

Lysaker, P. H., Erickson, M. A., Buck, B., Buck, K. D., Olesek, K., Grant, M. L., ... y Dimaggio, G. (2011). Metacognition and social function in schizophrenia: associations over a period of five months. Cognitive Neuropsychiatry, 16(3), 241-255. https://doi.org/ $10.1080 / 13546805.2010 .530470$

Lysaker, P. H., y Dimaggio, G. (2014a). Metacognitive Capacities for Reflection in Schizophrenia: Implications for developing treatments. Schizophrenia Bulletin, 40(3), 487491. https://doi.org/10.1093/schbul/sbu038 
Lysaker, P. H., Leonhardt, B.L., Pijnenborg, M., van Donkersgoed, R., de Jong, S., y Dimaggio, G. (2014b). Metacognition in Schizophrenia Spectrum Disorders: Methods of assessment and associations with neurocognition, symptoms, cognitive style and function. The Israel Journal of Psychiatry and Related Sciences, 51(1), 54-62. https://tinyurl.com/y9trlesw

Lysaker, P. H., Vohs, J., Minor, K. S., Irarrázaval, L., Leonhardt, B., Hamm, J. A., ... y Dimaggio, G. (2015). Metacognitive Deficits in Schizophrenia. Presence and associations with psychosocial outcomes. The Journal of Nervous and Mental Disease, 203(7), 530-536. https://doi.org/10.1097/NMD.0000000000000323

Lysaker, P. H., Buck, K., y Hamm, J. (2016). Escala de Evaluación de la Metacognición: Una breve visión general y Manual de Codificación para la versión abreviada (EEM-A) v 2015. Revista Gaceta de Psiquiatría Universitaria, 12(2), 174-190. https://tinyurl.com/y8d35gwj

Lysaker, P. H., Irarrázaval, L., Gagen, E. C., Armijo, I., Ballerini, M., Mancini, M., ... y Stanghellini, G.(2018). Metacognition in schizophrenia disorders: comparisons with community controls and bipolar disorder: replication with a Spanish language Chilean sample. Psychiatry Research 267, 528-534. https://doi.org/10.1016/j.psychres.2018.06.049

MacBeth, A., Gumley, A., Schwannauer, M., y Fisher, R. (2011). Attachment states of mind, mentalization, and their correlates in a first-episode psychosis sample. Psychology and Psychotherapy, 84(1), 42-57. https://doi.org/10.1348/147608310X530246

MacBeth, A., Gumley, A., Schwannauer, M., Carcione, A., Fisher, R., McLeod, H. J., y Dimaggio, G. (2014). Metacognition, symptoms and premorbid functioning in a first episode psychosis sample. Comprehensive Psychiatry, 55(2), 268-273. https://doi.org/10.1016/j.comppsych.2013.08.027

MacBeth, A., Gumley, A., Schwannauer, M., Carcione, A., McLeod, H.J., y Dimaggio, G. (2015). Metacognition in first episode psychosis: Item level analysis of associations with symptoms and engagement. Clinical Psychology and Psychotherapy, 23(4), 329-339. https://doi.org/10.1002/cpp.1959.

Main, M. (1991). Metacognitive knowledge, metacognitive monitoring, and singular (coherent) vs. multiple (incoherent) model of attachment. En C. Murray, J. Stevenson y P. Marris. (Eds.). Attachment across the life cycle. (pp 127-159). London, UK: Routledge

Ministerio de Salud. (2009). Guía Clínica para el tratamiento de personas desde primer episodio de Esquizofrenia. Guías Clínicas MINSAL. https://tinyurl.com/ybnsq4gk 
Nicolò, G., Dimaggio, G., Popolo, R., Carcione, A., Procacci, M., Hamm, J., ... y Lysaker, P. H. (2012). Associations of Metacognition with symptoms, isight and neurocognition in Schizophrenia in an Italian replication study. The Journal of Nervous and Mental Disease, 200(7), 644-647. https://doi.org/10.1097/NMD.0b013e31825bfb10

Peralta, V., y Cuesta, M. J. (1994). Psychometric properties of the positive and negative syndrome scale (PANSS) in schizophrenia. Psychiatry Research, 53(1), 31-40. https://doi.org/10.1016/0165-1781(94)90093-0.

Salvatore, G., Dimaggio, G., y Lysaker, P. H. (2007). An intersubjective perspective on negative symptoms of schizophrenia: implications of simulation theory. Cognitive Neuropsychiatry, 12(2), 144-164. https://doi.org/10.1080/13546800600819921

Salvatore, G., Dimaggio, G., Ottavi, P., y Popolo, R. (2017). Terapia Metacognitiva Interpersonale della Schizofrenia. La procedura formalizzata di intervento. Milán, Italia: Franco Angeli.

Semerari, A. (Ed). (2002). Psicoterapia cognitiva del paciente grave. Metacognición y relación terapéutica. Bilbao: Desclée de Brouwer.

Semerari, A., Carcione, A., Dimaggio, G., Falcone, M., Nicolo, G., Procacci, M., y Alleva, G. (2003). How to Evaluate Metacognitive Functioning in Psychotherapy? The Metacognition Assessment Scale and its Applications. Clinical Psychology and. Psychotherapy, 10(4), 238261. https://doi.org/10.1002/cpp.362

Tas, C., Brown E. C., Aydemir, O., Brüne, M., y Lysaker, P. H. (2014). Metacognition in Psychosis: Comparison of Schizophrenia with Bipolar Disorder. Psychiatry Research. 219(3), 464-469. https://doi.org/10.1016/j.psychres.2014.06.040

Trauelsen, A. M., Gumley, A., Jansen, J. E., Pedersen, M. B., Nielsen, H. L., Trier, C. H., ... Y Simonsen, E. (2016). Metacognition in first episode psychosis and its association with positive and negative symptom profiles. Psychiatry Research, 238, 14-23. https://doi.org/10.1016/j.psychres.2016.02.003

Van der Gaag, M., Hoffman, T., Remijsen, M., Hijman, R., De Haan, L., Van Meijel, B., ... Y Wiersma, D. (2006). The five factor model of the Positive and Negative Syndrome Scale II: A ten-fold cross-validation of a revised model. Schizophrenia Research, 85(1-3), 280-287. https://doi.org/10.1016/j.schres.2006.03.021

Vergara, S. (2018). Metacognición en Esquizofrenia: déficits metacognitivos y psicoterapia. Revista Chilena de Neuro-Psiquiatría, 56(4), 269-278. https://doi.org/10.4067/s071792272018000400269 
Vohs, J. L., Lysaker, P. H., Francis, M. M., Hamm, J., Buck, K. D., Olasek, K., ... y Breier, A. (2014). Metacognition, social cognition and symptoms in patients with first episode and prolonged psychosis. Schizophrenia Research, 153(1-3), 54-59. https://doi.org/10.1016/j.schres.2014.01.012

Vohs, J. L., George, S., Leonhardt, B. L., y Lysaker, P. H. (2016). An integrative model of the impairments in insight in schizophrenia: emerging research on causal factors and treatments. Expert Review of Neurotherapeutics, 16(10), 1193-1204. https://doi.org/ $10.1080 / 14737175.2016 .1199275$

\section{Para citar en APA}

Vergara-Ramírez, S. y León-Uribe, A. (2020). Metacognición, apego y sintomatología en esquizofrenia primer episodio. Terapia Psicologíca (En línea), 38(2), 131-152. https://doi.org/10.4067/ S0718-48082020000200131 